\title{
Correlation and familial aggregation of dimensions of psychosis in affected sibling pairs from China
}

Evangelos Vassos, Pak C. Sham, Guiqing Cai, Hong Deng, Xiehe Liu, Xueli Sun, Jinghua Zhao, Robin M. Murray, David A. Collier and Tao Li

\section{Background}

A number of studies with conflicting results have examined the familiality of schizophrenia syndromes in Western populations.
Aims
The objective of this study was to determine, using clinical data from concordant sibling pairs, whether symptom dimensions and other clinical characteristics of schizophrenia show familial aggregation and are therefore potentially useful traits in genetic studies.

\section{Method}
We measured clinical and demographic features, and symptom dimensions of schizophrenia in 137 families from china who had two or more affected members with schizophrenia. Within-sibling pair correlation was assessed with intraclass correlation coefficient and kappa statistics.

\section{Results}

Global functioning, positive, disorganisation and dysphoric symptoms, premorbid schizotypal and schizoid traits, premorbid social adjustment, type and age at illness onset all showed significant evidence of familial aggregation. DSM-IV schizophrenia subtypes were also found to be familial.

\section{Conclusions}

This is the first study in a large non-European population to confirm that schizophrenia dimensions and clinical characteristics show significant familiality, implying possible heritability. This supports their use in the delineation of homogeneous subsets for future genetic studies.

\section{Declaration of interest}

None. Funding detailed in Acknowledgements.
Since the foundations of the modern concepts of schizophrenia over the past 100 years, it has been considered divisible into symptom dimensions or various subtypes. ${ }^{1}$ These concepts have gradually progressed from a two-dimensional positive/negative symptom model $^{2,3}$ to multidimensional models of schizophrenia. ${ }^{4-8}$ The relationship between symptom dimensions or clinical characteristics and familial liability to schizophrenia has been measured in a series of studies, either to predict the occurrence of the disease or its spectrum disorders in the relatives of probands, with conflicting results. ${ }^{9-12}$

Five studies have examined within-family correlations of symptom dimensions in sibling pairs or families. Burke et $a l^{13}$ found modest correlation for positive, negative and disorganisation dimensions and Kendler et $a l^{14}$ found correlation for positive, negative and affective factors, whereas Loftus et $a l^{15}$ and Cardno et $a l^{16}$ detected significant within-sibling pair correlation only for disorganisation. Wickham et al concluded that psychomotor poverty, disorganisation and mania were familial.

All of the previous studies were conducted in Western industrialised populations, and it is thus important to examine people from other cultures and ancestry to investigate the familiality of psychotic symptoms and clinical characteristics. In addition, few studies have examined the intrafamiliar concordance of premorbid social adjustment and personality measures, like schizotypy. The aim of the present study is to determine the familiality of symptom dimensions in a sample of Han Chinese sibling pairs, with a view to using this information in future to explore the relationship between these dimensions and genetic susceptibility alleles as they are discovered.

\section{Method}

\section{Participants}

The participating families were ascertained as part of a project aimed at performing genetic linkage and association studies of psychosis in sibling pairs. In order to characterise the phenotype of these families, we decided to analyse the sample for siblingsibling correlation of symptom dimensions, subsequent to the clinical data collection process. The aim of this was to assess whether these dimensions would be useful phenotypic measures in molecular genetic analysis of the sample (i.e. whether they are likely to be genetic in origin).

One hundred and thirty-seven families, in which two or more siblings met DSM-IV ${ }^{17}$ criteria for schizophrenia, schizoaffective disorder or schizophreniform disorder, were recruited from Sichuan Province, Southwest China. Probands were identified from their hospital records or referrals by their psychiatrists, since their family history records showed they might have at least one sibling with a mental disorder. They were contacted at their home by one of the investigators, and agreed to take part in the study. The total number of families that were initially contacted was not recorded, so the refusal rate of participation is not known. The final sample consisted of 291 affected individuals (154 male, 137 female) from 137 families grouped as 122 sib-pairs, 14 triplets and 1 family with 5 affected siblings. In total, 5 parents ( 2 fathers, 3 mothers) had a diagnosis of schizophrenia. All of the participants were Han Chinese.

\section{Clinical and demographic assessment}

Age at examination, years of education and duration of illness in years were recorded. Age at onset was defined as the earliest age of psychotic symptoms or abnormal behaviours noticed by the individual or other people. Occupation was recorded as worker, agricultural worker, professional, self-employed or not working (including unemployed, housewife or househusband, etc.). Marriage was recorded as single, married, divorced or widowed.

All participants were interviewed in person by one of two psychiatrists using a Chinese version of the Structured Clinical Interview for DSM-IV Disorders (SCID) ${ }^{18}$ and the clinical scales described. The raters were not masked to sibling rating, i.e. before they conducted the interview, they knew that the participant had at least one sibling suffering from a mental disorder. Interviews 
were opportunistic, i.e. there were no systematic means of assigning a rater to a particular family, but instead this depended only on which psychiatrist was available to carry out the interview. Information was also taken from other sources including parental interview, case notes and contact with treating psychiatrists. The individual could be in any current stage of schizophrenia, i.e. first onset, remission or chronic. The two psychiatrists interviewed and rated the initial 36 families independently, which included a total of 144 people (affected siblings and parents if they were available), to assess the reliability of rating between them. The interrater reliability (kappa) between the two psychiatrists was $>0.80$ for all categorical items rated, and the intraclass correlation coefficient was $<0.70$ for continuous or ranked variables.

Diagnoses of schizophrenia, schizoaffective disorder or schizophreniform disorder were assigned on the basis of the interview and the medical records according to DSM-IV criteria. For all affected siblings in the sib-pair family sample, we also performed clinical symptom ratings using the Positive and Negative Symptom Scale (PANSS), ${ }^{19}$ on a lifetime worst basis. This scale consists of seven positive (PANSS-P) and seven negative (PANSS-N) symptom assessments and a 16-item general psychopathology assessment (PANSS-G), all rated on a 7-point severity scale. The PANSS has been validated in a Han Chinese population $^{20}$ and has been extensively used in samples with non-European ancestry. In addition, dimensions based on PANSS symptom scores were derived from an exploratory factor analysis.

The affected participants (and other informants when available) were asked to recall the symptoms during the most severe episode of their illness, and this was corroborated by clinical records, including hospital case notes. Lifetime worst rating was chosen as the most reliable measure of symptom dimensions in the sample, as individuals were at different stages of their illness when interviewed, and information on the most severe episode was most likely to have a written corroboration in the case notes. In addition, we measured aggressive behaviour with the Modified Overt Aggression Scale (MOAS) ${ }^{21}$ on a lifetime worst basis, premorbid social adjustment with the Premorbid Adjustment Scale (PAS), and Premorbid Schizoid and Schizotypal Traits (PSST). ${ }^{22}$ We used the Global Assessment of Function Scale $(\text { GAFS })^{23}$ to assess global functioning of participants on a lifetime worst basis. Premorbid Schizoid and Schizotypal Traits and PAS assessed the functional level of individuals during childhood and adolescence (5-16 years old).

\section{Statistical analyses}

Differences in demographic and clinical variables were examined with $t$-test and chi-squared test, as appropriate. Associations were tested with Pearson's correlation, or partial correlation to examine the relationship between two variables, while taking other variables into account. Before examining sibling resemblance, we tested the possible relationship of age, gender and duration of illness with the clinical variables. To remove the effect of age and gender, a linear regression model was fitted with age and gender as covariates and the residuals were used for post hoc analyses.

Extraction of factors from the PANSS symptom scores was performed with principal component analysis of correlation matrix. An initial factor analysis was performed with all PANSS items and the variables with a Kaiser-Meyer-Olkin measure $<0.5$ were dropped. The analysis was repeated and the number of meaningful factors was determined by the Scree plot. To aid interpretation of the solution, oblique rotation was performed by the promax method.

For within-sibling correlation analysis, all possible sib-pairs were formed from each family (e.g. each trio family was counted as three possible pairs, and the family with five affected siblings were counted as ten possible pairs). To correct for sibship size, each pair was weighted with a factor of $2 / n$ where $n$ is the number of affected members. Familiality was examined with kappa statistics or chi-squared test for categorical variables and intraclass correlation coefficient (ICC) for continuous variables. SPSS 13.0 program for Windows was used for all analyses. Bonferroni corrections for multiple testing were adopted, as appropriate.

\section{Results}

\section{General demographic and clinical variables}

The mean age at examination of the participants was 33.13 years and the mean number of years of education was 7.53. There was no significant difference in age at examination $(t=0.61, P=0.54)$ or occupation $\left(\chi^{2}=3.17, P=0.53\right)$ between men and women, but the men had spent longer in education $(t=2.3, P=0.022)$ and were more frequently unmarried $\left(\chi^{2}=24.53, P<0.0001\right)$. The distributions of general demographic variables are presented in Table 1. Years of education, occupation and marital status were significantly correlated within paired siblings (education: single measure ICC $=0.547, P<0.0001$; occupation: $\kappa=0.602, P<0.0001$; marriage: $\kappa=0.35, P<0.0005)$.

From the total of 137 families there were 174 pair-wise comparisons (i.e. three siblings count as three sib-pairs and five siblings as ten sib-pairs). The distribution of pairs by gender was 46 male-male, 36 female-female and 92 male-female. There was no excess of same gender pairs $\left(\chi^{2}=0.45\right.$, d.f. $\left.=1, P=0.503\right)$.

\begin{tabular}{|c|c|c|c|}
\hline Variable & Men $(n=154)$ & Women $(n=137)$ & Total $(n=291)$ \\
\hline Age at interview, years: mean (s.d.) & $33.42(8.68)$ & $32.80(8.66)$ & $33.13(8.66)$ \\
\hline Age at onset, years: mean (s.d.) & $23.22(6.55)$ & $23.18(7.53)$ & $23.20(7.02)$ \\
\hline Education, years: mean (s.d.) & $7.88(2.76)$ & $7.14(2.79)$ & $7.53(2.79)$ \\
\hline \multicolumn{4}{|l|}{ Occupation, $n(\%)$} \\
\hline Worker & $47(30)$ & $37(27)$ & $84(29)$ \\
\hline Agricultural worker & $70(45)$ & $62(45)$ & $132(45)$ \\
\hline Professional & $24(16)$ & $24(18)$ & $48(16)$ \\
\hline Self-employed & $2(1)$ & $6(4)$ & $8(3)$ \\
\hline Not working & $11(7)$ & $8(6)$ & $19(7)$ \\
\hline \multicolumn{4}{|l|}{ Marital status, $n(\%)$} \\
\hline Unmarried & $90(58)$ & $41(30)$ & $131(45)$ \\
\hline Married & $55(36)$ & $84(61)$ & $139(48)$ \\
\hline Divorced & $8(5)$ & $9(7)$ & $17(6)$ \\
\hline Widowed & $1(1)$ & $3(2)$ & $4(1)$ \\
\hline
\end{tabular}


The mean age at onset of psychosis was 23.20 years; the mean duration of illness was 9.93 years. There were no significant difference between males and females $(t=0.046, P=0.96)$ for the age at onset.

\section{Intercorrelations among demographic and clinical variables}

In order to assess if age at examination, gender and illness duration could be potential covariates for other related variables in this study, we performed a Pearson correlation with a number of variables, including clinical symptoms (PANSS-P, PANSS-N, PANSS-G and MOAS for aggressive behaviours), global assessment of function (GAFS), years of education, age at onset, onset type (acute, sub-acute, chronic) and premorbid characteristics (PAS and PSST). In general, age, gender and illness duration were not correlated with the variables above, with the following exceptions:

(a) age had weak correlation with positive symptoms $(r=0.173$, $P=0.003)$ and onset type $(r=0.178, P=0.002)$, and strong positive correlation with duration of illness $(r=0.584, P<0.0001)$ and age at onset $(r=0.487, P<0.0001)$;

(b) illness duration was positively correlated with PANSS-N $(r=0.185, \quad P=0.002$, using age at exam and gender as covariants), but not with other clinical variables.

Using affected siblings, we carried out partial correlation analysis between each of the psychotic symptom scales (PANSS-P, PANSS-N, PANSS-G and MOAS) and premorbid characteristics (PAS and PSST), with age at exam and gender as covariants. We found a positive correlation of PSST with PANSS-G ( $r=0.308$, $P<0.0001)$, PANSS $-\mathrm{N}(r=0.23, P<0.0005)$ and PAS $(r=0.295$, $P<0.0001)$. Premorbid social adjustment was positively correlated with PANSS-N $(r=0.248, P=0.0001)$.

\section{Factor structure of PANSS}

After the initial principal component analysis, only one item (grandiosity) had a Kaiser-Meyer-Olkin value $<0.5$ and was dropped. Four missing ratings in the total sample were replaced with the means. Eight factors had eigenvalues greater than unity and the Scree plot suggested a five-factor solution, in accordance with other studies employing factor analysis on PANSS. ${ }^{24-26}$ The Kaiser-Meyer-Olkin of sampling adequacy was 0.876 and the five-factor solution explained $60.75 \%$ of the total variance.

The first factor (negative) was aligned to the seven items of the PANSS-N and also to stereotyped thinking, social avoidance and poor attention of the general scale. Factor 2 (activation) composed of uncooperativeness, impulsivity, lack of judgement, hostility and excitement. Factor 3 was termed 'positive/disorganised' and included unusual thought content, disorientation, hallucinations and disturbance of volition. Factor 4 (dysphoric) had high loading for anxiety, somatic concern, depression, tension and guilt. Finally, factor 5 (so-called 'positive/delusional') was a second positive factor consisting of delusions, suspiciousness/persecution and hallucinations. The pattern matrix after promax rotation is presented in online Table DS1.

\section{Familiality of symptom dimensions and clinical characteristics}

From 174 pair-wise comparisons, 42 pairs were concordant for the paranoid subtype, 1 pair concordant for the disorganised subtype, 14 pairs concordant for catatonic subtype, 45 pairs concordant for undifferentiated subtype, 2 pairs concordant for schizophreniform disorder and 3 pairs concordant for schizoaffective disorder. The agreement of subtype within pairs was significant (Table 2) $(\kappa=0.43, P<0.0001)$.
Within 174 sib-pairs, the distribution of pairs by type at onset was as follows: acute-acute $=30$ (expected value $=15.6$ ); sub-acutesub-acute $=62$ (expected value $=55.5)$; chronic - chronic $=7$ (expected value $=3.1$ ). The agreement of type at onset within pairs was significant $(\kappa=0.251, P<0.0001)$. Age at onset and illness duration showed significant correlation within pairs (age at onset: single measure ICC $=0.346, P<0.0001$; illness duration: ICC $=0.349$, $P<0.0001)$.

Intraclass correlation coefficient analysis was performed to assess within-family correlations for global functioning, psychotic symptoms dimensions, aggression and premorbid characteristics. A Bonferroni correction for multiple testing was used and alpha was set at 0.006 . From the original scales used, significant within-family correlations between siblings were found for GAFS score, PANSS-P, PANSS-G, PSST and marginally for PAS, but not for PANSS-N and MOAS. Repetition of the analyses with the regression residuals, after adjustment for age and gender, gave similar results. As MOAS distribution departed substantially from normality, we repeated the analysis with Spearman's correlation, which gave similar results. The analysis of the five factors derived from PANSS produced highly significant within-family correlations for the positive/disorganised, dysphoric and positive/ delusional factors, but not for the negative and activation factors (Table 3).

\section{Discussion}

Schizophrenia is a highly heritable disorder, but the isolation of susceptibility genes has proved difficult. ${ }^{27,28}$ Reasons for this include locus heterogeneity, where different combinations of genes of small effect act to increase risk in each individual, and misclassification of the phenotype. ${ }^{29}$ One possible way to overcome the limitations on gene mapping is to refine the phenotype so that each individual susceptibility gene has a more direct relationship with a measurable characteristic, such as a quantitative score for a symptom dimension. For this to be successful, the symptom dimensions must have a heritable genetic basis, which can be explored through family, twin and adoption studies. Family studies cannot estimate the extent of heritability, but intrafamilial correlation between affected siblings or other relatives implies that a trait is influenced either by genes or shared environment. The latter is not thought to be important in schizophrenia. ${ }^{30}$ The identification of heritable quantitative traits may increase the power of genetic studies substantially.

In 137 families with two or more siblings affected by schizophrenia, using intraclass correlation coefficient analysis, we found that global functioning (measured by GAFS), two sub-scales of the PANSS (PANSS-P and PANSS-G, measuring positive symptoms and global psychopathology), three factors derived from PANSS

Table 2 Concordance of clinical subtype between sibling pairs, showing the observed and expected agreement ${ }^{a}$ Concordant subtypes Count, observed (expected)

\begin{tabular}{|lc|}
\hline Paranoid & $42(26.1)$ \\
\hline Disorganised & $1(0.023)$ \\
\hline Catatonic & $14(2.89)$ \\
\hline Undifferentiated & $45(27.3)$ \\
\hline Residual & $0(0.01)$ \\
\hline Schizoaffective disorder & $2(0.09)$ \\
\hline Schizophreniform disorder & $3(0.28)$ \\
\hline a. Overall within-pair correlation was significant at the $P<0.0001$ level.
\end{tabular}




\begin{tabular}{|c|c|c|c|c|}
\hline \multirow[b]{2}{*}{ Clinical variables } & \multirow[b]{2}{*}{ Weighted sib-pairs } & \multicolumn{3}{|c|}{ Intraclass correlation coefficient } \\
\hline & & $I C C^{a}$ & $P$ & $95 \% \mathrm{Cl}$ \\
\hline GAFS & 147 & 0.33 & 0.0002 & 0.18 to 0.47 \\
\hline MOAS & 152 & 0.03 & 0.36 & -0.13 to 0.19 \\
\hline \multicolumn{5}{|l|}{ PANSS } \\
\hline Positive & 152 & 0.36 & $<0.0001$ & 0.21 to 0.49 \\
\hline Negative & 152 & 0.05 & 0.256 & -0.10 to 0.21 \\
\hline General & 152 & 0.38 & $<0.0001$ & 0.24 to 0.51 \\
\hline PSST & 126 & 0.27 & 0.001 & 0.11 to 0.43 \\
\hline PAS & 126 & 0.21 & 0.006 & 0.04 to 0.36 \\
\hline \multicolumn{5}{|c|}{ Factors derived from PANSS } \\
\hline Negative & 146 & 0.06 & 0.235 & -0.10 to 0.22 \\
\hline Activation & 146 & 0.03 & 0.380 & -0.14 to 0.19 \\
\hline Positive/disorganised & 146 & 0.69 & $<0.0001$ & 0.59 to 0.76 \\
\hline Dysphoric & 146 & 0.35 & $<0.0001$ & 0.20 to 0.48 \\
\hline Positive/delusional & 146 & 0.43 & $<0.0001$ & 0.29 to 0.55 \\
\hline
\end{tabular}

(positive/disorganised, dysphoric and positive/delusional), premorbid schizotypy measured by PSST, and age at onset all showed evidence of familial aggregation. However, we did not find familial aggregation for either aggressive behaviour (MOAS) or negative symptoms (PANSS-N, i.e. blunted affect, emotional withdrawal, poor rapport, passive social withdrawal, difficulty in abstract thinking, lack of spontaneity and stereotyped thinking), negative and activation factors. The premorbid social adjustment (PAS) showed within sib-pair correlation of marginal significance after Bonferroni correction.

Our finding that positive symptoms correlate significantly between siblings contrasts with three previous studies of familial aggregation of symptom dimensions, which did not show strong familial aggregation for the positive dimension. ${ }^{7,15,16}$ However, two earlier studies ${ }^{13,14}$ showed that all the symptom factors (including positive) correlated in concordant siblings pairs. In addition, a study on the familiality of first-rank symptoms has showed that some of the nuclear symptoms (included in the positive sub-scale of PANSS) have a degree of familiality. ${ }^{31}$

Our study, in agreement with two previous studies ${ }^{15,16}$ failed to prove familial aggregation of the negative dimension. On the contrary, Wicham et al observed that psychomotor poverty exhibited the strongest familiality. In a recent study, comparing the severity of PANSS factors between individuals with schizophrenia with and without family history of schizophrenia, it was found that the negative factor was higher among individuals with a family history of schizophrenia. ${ }^{26}$ A smaller study, ${ }^{32}$ analysing negative symptoms in 39 individuals with schizophrenia and 38 controls, and each of their siblings, suggested that core negative symptoms of flattened affect and poverty of speech are not familial markers of liability, consistent with our results and those of others.

Previous family and twin studies ${ }^{15,16,33}$ indicate that disorganisation (positive formal thought disorder and/or inappropriate affect/bizarre behaviour) may be the most familial and thus most useful trait for genetic analysis. Our study differs in the clinical measures and traits evaluated, as the principal measure of subsyndromes was the PANSS. Although PANSS is qualitatively similar to the Scale for the Assessment of Positive Symptoms ${ }^{34}$ and the Scale for the Assessment of Negative Symptoms, ${ }^{35}$ it does not incorporate the items of inappropriate affect and bizarre behaviour, which usually load for the disorganised factor. The established sub-scales from the PANSS (positive symptoms, negative symptoms and global psychopathology) do not include a separate category for disorganisation. The conceptual disorganisation, disorientation and disturbance of volition (probably related at various degrees with the disorganised dimension) gave the stronger loading for the third factor, that was termed 'positive/ disorganised' because it incorporates also hallucinations and unusual thought content. Our findings of the strongest intrafamilial aggregation for the positive/disorganised factor may be partly accounted for by the strong familiality of the latent disorganisation dimension. Our study did not have significantly more power than previous studies, which analysed $109^{15}$ and $114^{16}$ sibling pairs and 61 mulitply affected families, ${ }^{7}$ compared with our data on 137 families.

In our study, we identified significant co-aggregation of same schizophrenia subtype within families. This is in agreement with the observation by Kendler et $a l^{14}$ that latent classes correlated within sib-pairs, but contrary to the results by Cardno et al, ${ }^{34}$ who found no intrafamiliar association of subtypes. The two studies differ in that we used subtypes according to DSM-IV, whereas Cardno et al allocated their participants to subtypes using criteria from literature-based taxonomies.

Age at onset, illness severity measured with GAFS, type at onset and duration of illness were found to correlate significantly within sibling pairs, as suggested by the majority of previous studies. ${ }^{13,14,36-38}$ Our observation that premorbid schizoid and schizotypal traits are significantly correlated between sib-pairs is in agreement with studies indicating that schizotypy is a familial trait. $^{39,40}$

Poor premorbid social adjustment appears to be correlated with more severe premorbid schizoid and schizotypal traits and more severe negative symptoms. This is in agreement with Wickham et al, ${ }^{7}$ who found that psychomotor poverty was significantly related to poor premorbid functioning. In our sample, more severe premorbid schizoid and schizotypal traits were associated with more negative and general psychopathological symptoms, and there was a trend for less positive symptoms. Illness duration was only associated with negative symptoms. It is likely that premorbid social adjustment may be an expression of some deficit of early neurodevelopment, compatible with the view of schizophrenia proposed by van Os et $a l,{ }^{41}$ who suggested that chronic negative cases are predicted by neurodevelopmental impairment.

A number of studies have shown an earlier age at onset, poorer premorbid psychosocial adjustment and greater risk for relapse 
among men. Interestingly, in our sample of 291 Han Chinese people with schizophrenia, we found no difference between men and women regarding age at onset, premorbid psychosocial adjustment and clinical performance.

There are a number of limitations of this study. The participating families were not ascertained epidemiologically but as part of a molecular genetic study and may therefore be subject to ascertainment bias such as the selection of more severe cases because psychiatric hospital case records were used for recruitment. The raters were masked to the purpose of this study, but the same rater interviewed both siblings so was not masked to the data on the first sibling when interviewing the second. This might have inflated within-family correlations. To minimise this problem, several cases were rated by both interviewers and a good interrater reliability was established. In addition, there was no systematic assignment of raters to families, but interviews were instead performed on an opportunistic basis. These factors could potentially affect sibling correlations. We have performed a number of statistical tests, and so caution in interpreting $P$ values is required. We have adopted a conservative Bonferroni adjustment to $P$ values to control for this.

As in most other genetic studies of psychosis, the data in the study was not collected prospectively and this may underestimate the occurrence of psychotic symptoms, reducing the evidence for sibling correlation. By rating worse episode symptoms it is possible that negative symptom severity were less accurately recorded because they are possibly less memorable and this may have affected the familiality estimates of the negative factor. For this reason, clinical examination was corroborated by case notes and interviews with treating psychiatrists. In addition, negative symptoms, though less memorable, are more enduring and can be detected even subsequent to an episode of psychosis. The retrospective rating of premorbid adjustment and personality may have been affected by memory biases or symptoms present at the time of the interview. Results concerning premorbid traits should be interpreted in the light of this limitation.

It is possible that the sibling correlations we observed arose from genetic variance in traits that are not directly related to schizophrenia liability (siblings are genetically similar and will tend to be concordant for a wide range of traits of all types). However, the traits and symptoms we examined are characteristic of schizophrenia and thus likely to be aetiologically important. In spite of these limitations, this study offers evidence that several dimensions of psychosis show strong intrafamilial correlation in a sample of non-European individuals. The findings can be used for more accurate characterisation of the phenotype in future genetic studies in Asian populations.

\footnotetext{
Evangelos Vassos, MD, PhD, King's College London, Institute of Psychiatry, Division of Psychological Medicine \& Psychiatry, and Social, Genetic \& Developmental Psychiatry Research Centre, London, UK; Pak C. Sham, MD, PhD, MRCPsych, King's College London, Institute of Psychiatry, Division of Psychological Medicine \& Psychiatry, and Social, Genetic \& Developmental Psychiatry Research Centre, London, UK, and Department of Psychiatry, LKS Faculty of Medicine, The University of Hong Kong, China; Guiqing Cai, MD, Hong Deng, MD, Xiehe Liu, MD, Xueli Sun, MD, The Psychiatric Laboratory and Department of Psychiatry, West China Hospital, Sichuan University, China; Jinghua Zhao, PhD, Robin M. Murray, MD, DSC, FRCPsych, King's College London, Institute of Psychiatry, Division of Psychological Medicine and Psychiatry, London, UK; David A. Collier, PhD, King's College London, Institute of Psychiatry, Division of Psychological Medicine \& Psychiatry, and Social, Genetic \& Developmental Psychiatry Research Centre, London, UK; Tao Li, MD, PhD, King's College London, Institute of Psychiatry, Division of Psychological Medicine \& Psychiatry, and Social, Genetic \& Developmental Psychiatry Research Centre, London UK, and The Psychiatric Laboratory and Department of Psychiatry, and State Key Laboratory of Biotherapy, West China Hospital, Sichuan University, China
}

Correspondence: Tao Li, Institute of Psychiatry, Box P082, De Crespigny Park Denmark Hill, London SE5 8AF, UK. Email: tao.li@iop.kcl.ac.uk

First received 5 Oct 2007, final revision 22 Apr 2008, accepted 16 May 2008

\section{Acknowledgements}

This work was partly funded by the National Nature Science Foundation of China (30530300 and 30125014) (T.L.), NARSAD Independent Investigator Award (T.L.), the Wellcome Trust (International Collaborative award to T.L., D.A.C., X.S. and X.L.), the National Basic Research Program of China (973 Program 2007CB512301) (T.L.), European Commission (SGENE) (D.A.C., E.V., T.L.) and the State Scholarship Foundation of Greece (E.V.).

\section{References}

1 Adityanjee, Aderibigbe YA, Theodoridis D, Vieweg VR. Dementia praecox to schizophrenia: the first 100 years. Psychiatry Clin Neurosci 1999; 53: 437-48.

2 Crow TJ. Molecular pathology of schizophrenia: more than one disease process? BMJ 1980; 280: 66-8.

3 Andreasen NC, Olsen S. Negative $v$. positive schizophrenia. Definition and validation. Arch Gen Psychiatry 1982; 39: 789-94.

4 Liddle PF. The symptoms of chronic schizophrenia. A re-examination of the positive-negative dichotomy. Br J Psychiatry 1987; 151: 145-51.

5 Andreasen NC. Positive vs. negative schizophrenia: a critical evaluation. Schizophr Bull 1985; 11: 380-9.

6 Peralta V, Cuesta MJ, de Leon J. An empirical analysis of latent structures underlying schizophrenic symptoms: a four-syndrome model. Biol Psychiatry 1994; 36: 726-36.

7 Wickham H, Walsh C, Asherson P, Taylor C, Sigmundson T, Gill M, Owen MJ, McGuffin P, Murray R, Sham P. Familiality of symptom dimensions in schizophrenia. Schizophr Res 2001; 47: 223-32.

8 Dikeos DG, Wickham H, McDonald C, Walshe M, Sigmundsson T, Bramon E, Grech A, Toulopoulou T, Murray R, Sham PC. Distribution of symptom dimensions across Kraepelinian divisions. Br J Psychiatry 2006; 189: 346-53.

9 Kendler KS, McGuire M, Gruenberg AM, Walsh D. Clinical heterogeneity in schizophrenia and the pattern of psychopathology in relatives: results from an epidemiologically based family study. Acta Psychiatr Scand 1994; 89: 294-300.

10 Cardno AG, Holmans PA, Harvey I, Williams MB, Owen MJ, McGuffin P. Factor-derived subsyndromes of schizophrenia and familial morbid risks. Schizophr Res 1997; 23: 231-8.

11 van Os J, Marcelis M, Sham $\mathrm{P}$, Jones $\mathrm{P}$, Gilvarry $\mathrm{K}$, Murray R. Psychopathological syndromes and familial morbid risk of psychosis. $\mathrm{Br} J$ Psychiatry 1997; 170: 241-6.

12 Peralta V, Cuesta MJ. The relationship between syndromes of the psychotic illness and familial liability to schizophrenia and major mood disorders. Schizophr Res 2007; 91: 200-9.

13 Burke JG, Murphy BM, Bray JC, Walsh D, Kendler KS. Clinical similarities in siblings with schizophrenia. Am J Med Genet 1996; 67: 239-43.

14 Kendler KS, Karkowski-Shuman L, O'Neill FA, Straub RE, MacLean CJ, Walsh D. Resemblance of psychotic symptoms and syndromes in affected sibling pairs from the Irish Study of High-Density Schizophrenia Families: evidence for possible etiologic heterogeneity. Am J Psychiatry 1997; 154: 191-8.

15 Loftus J, DeLisi LE, Crow TJ. Familial associations of subsyndromes of psychosis in affected sibling pairs with schizophrenia and schizoaffective disorder. Psychiatry Res 1998; 80: 101-11.

16 Cardno AG, Jones LA, Murphy KC, Sanders RD, Asherson P, Owen MJ, McGuffin P. Dimensions of psychosis in affected sibling pairs. Schizophr Bull 1999; 25: 841-50.

17 American Psychiatric Assocation. Diagnostic and Statistical Manual of Mental Disorders (4th edn) (DSM-IV). APA 1994.

18 First MB, Gibbon M, Spitzer RL, Williams LBW. User Guide for the Structured Clinical Interview for DSM-IV-R Axis I Disorders (Research Version). Biometrics Research, 2001.

19 Kay SR, Fiszbein A, Opler LA. The positive and negative syndrome scale (PANSS) for schizophrenia. Schizophr Bull 1987; 13: 261-76.

20 Cheng JJ HH, Chang CJ, Lane HY, Hu HG. Positive and negative syndrome scale (PANSS): establishment and reliability study of a mandarin Chinese language version. Chinese Psychiatry 1996; 10: 251-8.

21 Kay SR, Wolkenfeld F, Murrill LM. Profiles of aggression among psychiatric patients. I. Nature and prevalence. J Nerv Ment Dis 1988; 176: 539-46.

22 Foerster A, Lewis S, Owen M, Murray R. Pre-morbid adjustment and personality in psychosis. Effects of sex and diagnosis. Br J Psychiatry 1991; 158: $171-6$.

23 Goldman $\mathrm{HH}$, Skodol AE, Lave TR. Revising axis V for DSM-IV: a review of measures of social functioning. Am J Psychiatry 1992; 149: 1148-56.

24 White L, Harvey PD, Opler L, Lindenmayer JP. Empirical assessment of the factorial structure of clinical symptoms in schizophrenia. A multisite, multimodel evaluation of the factorial structure of the Positive and Negative 
Syndrome Scale. The PANSS Study Group. Psychopathology 1997; 30: 263-74.

25 Malaspina D, Goetz RR, Yale S, Berman A, Friedman JH, Tremeau F, Printz D, Amador X, Johnson J, Brown A, Gorman JM. Relation of familial schizophrenia to negative symptoms but not to the deficit syndrome. Am J Psychiatry 2000; 157: 994-1003.

26 Ritsner M, Ratner Y, Gibel A, Weizman R. Familiality in a five-factor model of schizophrenia psychopathology: findings from a 16-month follow-up study. Psychiatry Res 2005; 136: 173-9.

27 Riley BP, McGuffin P. Linkage and associated studies of schizophrenia. Am J Med Genet 2000; 97: 23-44.

28 Crow TJ. How and why genetic linkage has not solved the problem of psychosis: review and hypothesis. Am J Psychiatry 2007; 164: 13-21.

29 Tabor HK, Risch NJ, Myers RM. Candidate-gene approaches for studying complex genetic traits: practical considerations. Nat Rev Genet 2002; 3: 391-7.

30 Cardno AG, Gottesman, II. Twin studies of schizophrenia: from bow-andarrow concordances to star wars $\mathrm{Mx}$ and functional genomics. Am J Med Genet 2000; 97: 12-7.

31 Loftus J, Delisi LE, Crow TJ. Factor structure and familiality of first-rank symptoms in sibling pairs with schizophrenia and schizoaffective disorder. Br J Psychiatry 2000; 177: 15-9.

32 Craver JC, Pogue-Geile MF. Familial liability to schizophrenia: a sibling study of negative symptoms. Schizophr Bull 1999; 25: 827-39.

33 Cardno AG, Sham PC, Murray RM, McGuffin P. Twin study of symptom dimensions in psychoses. Br J Psychiatry 2001; 179: 39-45.
34 Andreasen N. Scale for the Assessment of Positive Symptoms (SAPS). University of lowa, 1984.

35 Andreasen N. Scale for the Assessment of Negative Symptoms (SANS). Univeristy of lowa, 1984.

36 Cardno AG, Jones LA, Murphy KC, Sanders RD, Asherson P, Owen MJ, McGuffin P. Sibling pairs with schizophrenia or schizoaffective disorder: associations of subtypes, symptoms and demographic variables. Psycho Med 1998; 28: 815-23.

37 Wickham $\mathrm{H}$, Walsh $\mathrm{C}$, Asherson $\mathrm{P}$, Gill M, Owen MJ, McGuffin P, Murray R, Sham P. Familiality of clinical characteristics in schizophrenia. J Psychiatr Res 2002; 36: 325-9.

38 DeLisi LE, Goldin LR, Maxwell ME, Kazuba DM, Gershon ES. Clinical features of illness in siblings with schizophrenia or schizoaffective disorder. Arch Gen Psychiatry 1987; 44: 891-6.

39 Kendler KS, McGuire M, Gruenberg AM, Walsh D. Schizotypal symptoms and signs in the Roscommon Family Study. Their factor structure and familial relationship with psychotic and affective disorders. Arch Gen Psychiatry 1995: 52: 296-303.

40 Mata I, Sham PC, Gilvarry CM, Jones PB, Lewis SW, Murray RM. Childhood schizotypy and positive symptoms in schizophrenic patients predict schizotypy in relatives. Schizophr Res 2000; 44: 129-36.

41 van Os J, Fahy TA, Jones $P$, Harvey I, Sham $P$, Lewis S, Bebbington $P$, Toone $B$, Williams M, Murray R. Psychopathological syndromes in the functional psychoses: associations with course and outcome. Psychol Med 1996; 26 $161-76$ 